\title{
Einladung zum Intensivkurs Muskuloskelettale Radiologie in Würzburg
}

Sehr geehrte Kolleginnen und Kollegen,

es ist uns eine große Freude, Sie im „Röntgenjahr 2020“ nach Würzburg an den Ort der Entdeckung der Röntgenstrahlen zum Intensivkurs Muskuloskelettale Radiologie (IMR) einzuladen.

Die Radiographie der Hand von Wilhelm Conrad Röntgens Ehefrau Anna Bertha ist Ihnen allen gut bekannt. Diese erste muskuloskelettale Anwendung der „neuen Art von Strahlen“ nutzte Röntgen in seinem Forschungslabor in Würzburg. 125 Jahre nach dieser für unser Fach so bedeutenden Entdeckung der Röntgenstrahlen haben wir den erfolgreichen Intensivkurs Muskuloskelettale Radiologie aus Hamburg auch nach Würzburg geholt, und dürfen Sie zu einem zweitägigen Fortbildungsseminar in die traditionsreiche Stadt einladen.

Der Intensivkurs Muskuloskelettale Radiologie bietet Ihnen eine ausgewogene Balance zwischen praxisrelevantem Basiswissen, Protokollempfehlungen für die tägliche Routine und innovativem Spezialwissen. Für dieses Programm konnten wir weithin bekannte und hochkarätige Referentinnen und Referenten gewinnen.

In zahlreichen halbstündigen Präsentationen von Expertinnen und Experten aus Deutschland und Österreich werden Sie Gelegenheit haben, Ihre Kenntnisse in unterschiedlichen Teilgebieten der muskuloskelettalen Diagnostik aufzufrischen und zu vertiefen. Die Vorträge vermitteln Basis- und Expertenwissen mit aktuellen Erkenntnissen zum Schulter-, Hand- und Kniegelenk. Zusätzlich werden Sie Schwerpunktsitzungen zur Bildgebung von Knochentumoren und zur Bildgebung rheumatisch-entzündlicher Erkrankungen des muskuloskelettalen Systems erleben.

Im großzügigen Hörsaal des Zentrums für Operative Medizin (ZOM) des Universitätsklinikums Würzburg werden klinische Grundlagen, Schnittbildanatomie und Untersu-

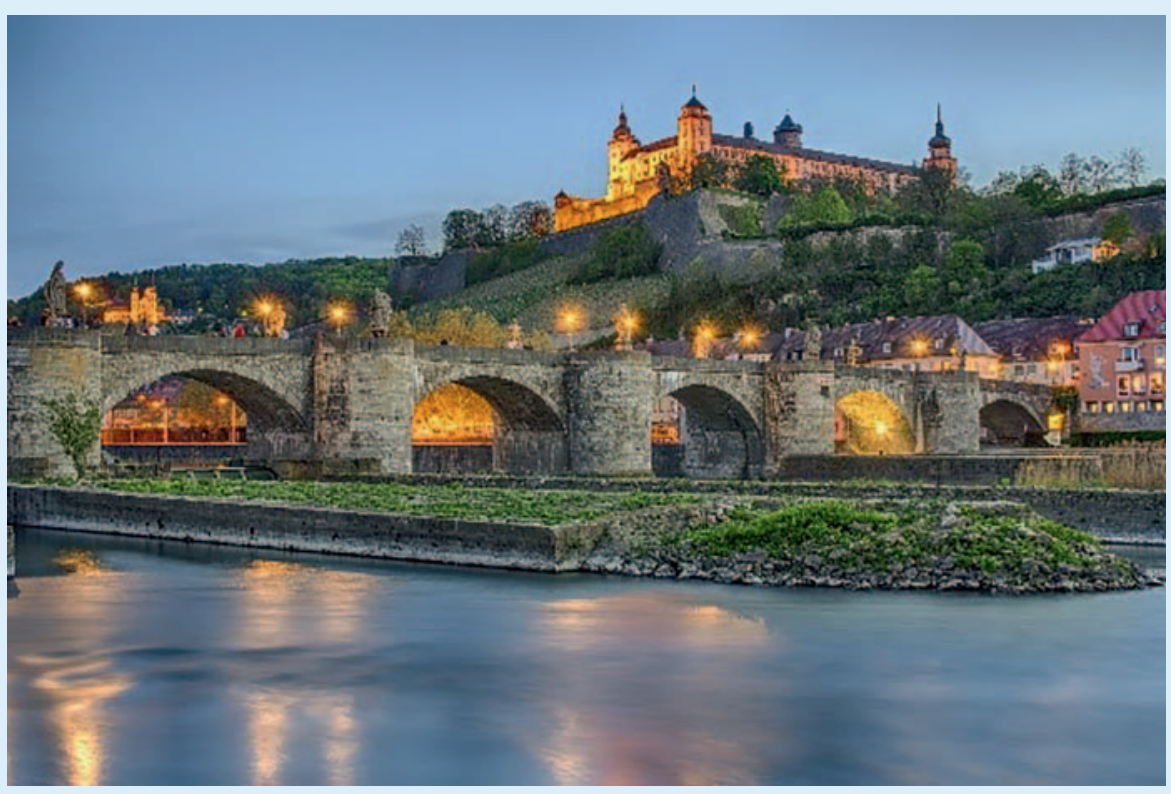

Der IMR findet 2020 passend zum Röntgenjahr auch in Würzburg statt.

chungstechnik erörtert, bildmorphologische Korrelate präsentiert sowie häufige und seltene Differentialdiagnosen strukturiert vorgestellt.

Der IMR soll Ihnen auch die Gelegenheit zum intensiven Austausch zwischen Teilnehmenden sowie Referentinnen und Referenten bieten. Die im Kurs erworbenen CME-Punkte werden für die „Zertifizierung zum Experten der Muskuloskelettalen Radiologie“ der Deutschen Röntgengesellschaft anerkannt.

Neben Fortbildung und Wissensaustausch soll die Geselligkeit nicht zu kurz kommen. Nach Abschluss des ersten Tages laden wir Sie zum Gesellschaftsabend in den ehrwürdigen Gartenpavillion des Juliusspitals ein. Schon Rudolf Virchow hatte in diesen damals „Theatrum Anatomicum" genannnten barocken Gebäude seine Studien durchgeführt.

Wir freuen uns auf den regen Austausch mit Ihnen und wünschen Ihnen eine interessante und ereignisreiche Tagung in Würzburg.

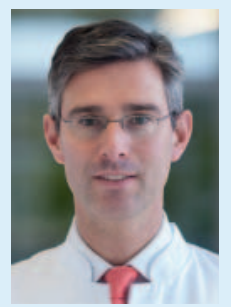

Prof. Dr. med. Thorsten Bley
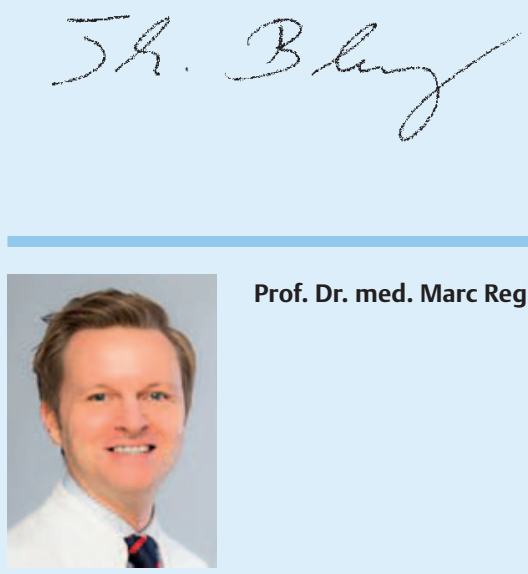

Prof. Dr. med. Marc Regier

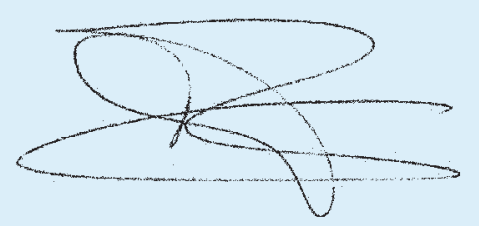

